\title{
BOUND STATES FOR RAPIDLY OSCILLATORY SCHRÖDINGER OPERATORS IN DIMENSION 2
}

\author{
ALEXIS DROUOT
}

\begin{abstract}
We study the eigenvalues of Schrödinger operators $-\Delta_{\mathbb{R}^{2}}+V_{\varepsilon}$ on $\mathbb{R}^{2}$ with rapidly oscillatory potential $V_{\varepsilon}(x)=W(x, x / \varepsilon)$, where $W(x, y) \in C_{0}^{\infty}\left(\mathbb{R}^{2} \times \mathbb{T}^{2}\right)$ satisfies $\int_{\mathbb{T}^{2}} W(x, y) d y=0$. We show that for $\varepsilon$ small enough, such operators have a unique negative eigenvalue, that is exponentially close to 0 .
\end{abstract}

\section{INTRODUCTION.}

We study the $L^{2}$-eigenvalues of the Schrödinger operator $-\Delta_{\mathbb{R}^{2}}+V_{\varepsilon}$ on $\mathbb{R}^{2}$, where $\varepsilon$ is a small parameter and $V_{\varepsilon}$ is a real-valued, compactly supported, and rapidly oscillatory potential on $\mathbb{R}^{2}$ :

$$
V_{\varepsilon}(x)=W(x, x / \varepsilon), \quad W(x, y)=\sum_{k \in \mathbb{Z}^{2} \backslash 0} W_{k}(x) e^{i k y} .
$$

The functions $W_{k}$ are smooth, with support in $\mathbb{B}(0, L)=\left\{x \in \mathbb{R}^{2},|x|<L\right\}$, and satisfy $\overline{W_{k}}=W_{-k}$. The potential $V_{\varepsilon}$ is a first approximation to model disordered medias with scale of heterogeneity $\sim \varepsilon$ - oscillations play here the role of randomness. This note provides a simple proof of a conjecture of Duchêne-Vukićević-Weinstein [DVW14]:

Theorem 1. For $\varepsilon$ small enough, the operator $-\Delta_{\mathbb{R}^{2}}+V_{\varepsilon}$ has a unique bound state, with energy $E_{\varepsilon}$ given by

$$
E_{\varepsilon}=-\exp \left(-\frac{4 \pi}{\varepsilon^{2} \int_{\mathbb{R}^{2}} \Lambda_{0}(x) d x+o\left(\varepsilon^{2}\right)}\right), \quad \Lambda_{0}(x) \stackrel{\text { def }}{=} \sum_{k \neq 0} \frac{\left|W_{k}(x)\right|^{2}}{|k|^{2}} .
$$

In one dimension, the study of the spectral properties of $-\partial_{x}^{2}+V_{\varepsilon}$ with $V_{\varepsilon}$ rapidly oscillatory originated with Borisov-Gadyl'shin [BoGa06], who gave a sufficient condition for the existence of a bound state, and derived the asymptotic of the corresponding energy:

$$
E_{\varepsilon}=-\frac{\varepsilon^{4}}{4} \int_{\mathbb{R}} \Lambda_{0}(x) d x, \quad \Lambda_{0}(x) \stackrel{\text { def }}{=} \sum_{k \neq 0} \frac{\left|W_{k}(x)\right|^{2}}{|k|^{2}} .
$$

This study was continued later in Borisov [Bo07] then Duchêne-Weinstein [DW11], in particular to include less regular potentials.

Duchêne-Vukićević-Weinstein [DVW14] studied the behavior of scattering quantities of Schrödinger operators with potentials which are the sum of a slowly varying term

Date: September 22, 2021. 
$W_{0}$ and a rapidly oscillatory term $V_{\varepsilon}$. They showed that the transmission coefficient of the effective potential $W_{0}-\varepsilon^{2} \Lambda_{0}$, which is a $O\left(\varepsilon^{2}\right)$-perturbation of $W_{0}$, differs from the one of $W_{0}+V_{\varepsilon}$ by $O\left(\varepsilon^{3}\right)$. When $W_{0}=0$, they recovered the result of [BoGa06]: $-\partial_{x}^{2}+V_{\varepsilon}$ has a unique negative eigenvalue satisfying (1.2) for small $\varepsilon$. They proved a uniform weighted dispersive estimate for the propagator $e^{i t\left(-\partial_{x}^{2}+V_{\varepsilon}\right)}$ as $\varepsilon \rightarrow 0$, despite the presence of an eigenvalue near the edge of the continuous spectrum.

Recently, Duchêne-Raymond [DR16] obtained homogenization results for potentials of the form $\varepsilon^{-\beta} V_{\varepsilon}, \beta \in(0,2)$, in dimension 1 , using a normal form approach. Dimassi [Di16] applied $\varepsilon$-semiclassical calculus to show a trace formula and a Weyl law for potentials of the form $\varepsilon^{-2} V_{\varepsilon}$, in any dimension $d$.

Motivated by [DVW14] and by Christiansen [Ch06], we used a different approach to study in [Dr15] the resonances and eigenvalues of $-\Delta_{\mathbb{R}^{d}}+W_{0}+V_{\varepsilon}$, in any odd dimension $d$. When $W_{0}=0$, we proved that the resonances and eigenvalues of $V_{\varepsilon}$ escape all bounded regions as $\varepsilon \rightarrow 0$ (except the one converging to 0 when $d=1$ ). When $W_{0} \neq 0$, we showed that the resonances and eigenvalues of $W_{0}+V_{\varepsilon}$ converge to the one of $W_{0}$, with a complete expansion in powers of $\varepsilon$. We improved upon the homogeneization result of [DVW14], refining the effective potential $W_{0}-\varepsilon^{2} \Lambda_{0}$ to $W_{0}-\varepsilon^{2} \Lambda_{0}-\varepsilon^{3} \Lambda_{1}$, and deriving it for any odd $d$. We refer to [Dr15, Figure 2] for numerical results and to [Dr15, §1] for additional references.

A famous result of Simon [Si76, Theorem 3.4] in dimension 2 predicts that under suitable conditions, a Schrödinger operator with small negative potential $-\Delta_{\mathbb{R}^{2}}+\epsilon \Lambda$ has a unique bound state with energy

$$
E_{\varepsilon}=-\exp \left(-\frac{4 \pi}{\epsilon \int_{\mathbb{R}^{2}} \Lambda(x) d x+o(\epsilon)}\right) .
$$

This identity put together with (1.1) supports the main idea of [DVW14]: the scattering quantities of rapidly oscillatory potentials are similar to the one of suitable small potentials.

To prove Theorem 1, we first follow [Si76, §3]: we use a modified Fredholm determinant to reduce the study of eigenvalues of $-\Delta_{\mathbb{R}^{2}}+V_{\varepsilon}$ to an equation involving a certain trace. Then, we provide estimates on this trace following some ideas of [Dr15], ending the proof. We mention that the result of Theorem 1 still applies when $W$ is not smooth but satisfies instead the weaker bound

$$
\sum_{k \neq 0}\left|W_{k}\right|_{\infty}+\frac{\left|W_{k}\right|_{C^{1}}}{|k|}+\frac{\left|W_{k}\right|_{C^{2}}}{|k|^{2}}+\frac{\left|W_{k}\right|_{C^{3}}}{|k|^{3}}+\sum_{0 \neq k \neq \ell} \frac{\left|W_{k}\right|_{C^{3}}\left|W_{\ell}\right|_{C^{3}}}{|k-\ell|^{5 / 2}}<\infty
$$

with no change in the proof.

Aknowledgement. We would like to thanks Maciej Zworski for valuable discussions. This research was supported by the NSF grant DMS-1500852 and the Fondation CFM pour la recherche.

Notations. We will use the following: 
- For $x \in \mathbb{R}^{2},\langle x\rangle$ denotes the Japanese bracket of $x:\langle x\rangle \stackrel{\text { def }}{=}\left(1+|x|^{2}\right)^{1 / 2}$.

- The function $z \mapsto \ln (z)$ denotes the holomorphic logarithm on $\mathbb{C} \backslash(-\infty, 0]$.

- $C_{0}^{\infty}\left(\mathbb{R}^{2}, \mathbb{R}\right)$ is the set of real smooth compactly supported functions on $\mathbb{R}^{2}$.

- $L^{2}$ denotes the Hilbert space of functions that are squared integrable, with $L^{2}$-norm denoted by $|f|_{2}$ and scalar product given by $\langle f, g\rangle_{2}=\int_{\mathbb{R}^{2}} \bar{f} g$.

- $C^{k}$ is the space of functions on $\mathbb{R}^{2}$ with $k$ continuous and bounded derivatives, with norm $|f|_{C^{k}}=|f|_{\infty}+\left|f^{\prime}\right|_{\infty}+\ldots+\left|f^{(k)}\right|_{\infty}$.

- We write $\Delta$ for the Laplacian $\Delta_{\mathbb{R}^{2}}$, and $\hat{f}$ for the Fourier transform of $f$ : $\hat{f}(\xi)=\frac{1}{2 \pi} \int_{\mathbb{R}^{2}} e^{-i x \xi} f(x) d x$. $H^{s}$ denotes the standard Sobolev space on $\mathbb{R}^{2}$, with norm $|f|_{H^{s}}=\left|\langle\xi\rangle^{s} \hat{f}\right|_{2}=\left|(\operatorname{Id}-\Delta)^{s / 2} f\right|_{2}$.

- $\mathscr{B}$ is the Banach space of bounded linear operators from $L^{2}$ to itself.

- $\mathscr{L}^{2}$ is the Banach space of Hilbert-Schmidt operators on $L^{2}$.

\section{General properties.}

Let $R_{0}(\lambda, x, y)$ be the kernel of the free resolvent $\left(-\Delta+\lambda^{2}\right)^{-1}$. It its a Hankel function of $\lambda|x-y|$ and one can write

$$
R_{0}(\lambda, x, y)=-\frac{1}{2 \pi} \ln (\lambda)+H_{0}(\lambda, x, y),
$$

where for every $x \neq y$ the function $\lambda \mapsto H_{0}(\lambda, x, y)$ is holomorphic in $\{\operatorname{Re} \lambda>0\}$ and admits a continuous extension to $\{\operatorname{Re} \lambda \geq 0\}-$ see $[\operatorname{Si76},(12)]$. For $\lambda$ with $\operatorname{Re} \lambda \geq 0$, let $H_{0}(\lambda)$ be the operator with kernel $H_{0}(\lambda, x, y)$. When $\lambda \in(0, \infty), R_{0}(\lambda)$ is selfadjoint and $\ln (\lambda)$ is real, hence $H_{0}(\lambda)$ is selfadjoint.

Let $\mathscr{V} \in C_{0}^{\infty}\left(\mathbb{R}^{2}, \mathbb{R}\right)$, with support in $\mathbb{B}(0, L)=\left\{x \in \mathbb{R}^{2},|x| \leq L\right\}$, and $\rho \in$ $C_{0}^{\infty}\left(\mathbb{R}^{2}, \mathbb{R}\right)$, equal to 1 on the support of $\mathscr{V}$ and 0 outside $\mathbb{B}(0, L)$. We will be interested in the behavior of $K_{\mathscr{V}}(\lambda)=\rho R_{0}(\lambda) \mathscr{V}$ for $\lambda$ close to 0 . Write $K_{\mathscr{V}}(\lambda)=\Pi_{\mathscr{V}} \ln (\lambda)+L_{\mathscr{V}}(\lambda)$,

$$
\Pi_{\mathscr{V}} \stackrel{\text { def }}{=}-\frac{1}{2 \pi} \rho \otimes \mathscr{V}, \quad L_{\mathscr{V}}(\lambda) \stackrel{\text { def }}{=} \rho H_{0}(\lambda) \mathscr{V}, \quad \operatorname{Re} \lambda \geq 0 .
$$

An operator $A: C_{0}^{\infty}\left(\mathbb{R}^{2}\right) \rightarrow \mathscr{D}^{\prime}\left(\mathbb{R}^{2}\right)$ belongs to $\mathscr{L}^{2}$ (the Hilbert-Schmidt class) if and only if its kernel $A(x, y)$ is in $L^{2}\left(\mathbb{R}^{4}, d x d y\right)$. In this case the $\mathscr{L}^{2}$-norm is

$$
|A| \mathscr{L}^{2} \stackrel{\text { def }}{=}\left(\int_{\mathbb{R}^{2} \times \mathbb{R}^{2}}|A(x, y)|^{2} d x d y\right)^{1 / 2} .
$$

We prove the following result, similar to [Si76, Proposition 3.2]:

Lemma 2.1. The operator $L_{\rho}(\lambda)$ is in $\mathscr{L}^{2}$. Moreover, uniformly locally in $\lambda, \mu$ with nonnegative real parts,

$$
\left|L_{\rho}(\lambda)-L_{\rho}(\mu)\right|_{\mathscr{B}}=O(|\lambda \ln (\lambda)-\mu \ln (\mu)|), \quad\left|L_{\rho}(\lambda)\right|_{L^{2} \rightarrow H^{2}}=O(1) .
$$

Proof. To prove the first part of (2.1), we write the kernel of $H_{0}(\lambda)$ as

$$
H_{0}(\lambda, x, y)=-\frac{1}{2 \pi} \ln |x-y|+F(\lambda|x-y|) .
$$


The function $F: \mathbb{C} \rightarrow \mathbb{C}$ takes the form $F(\zeta)=\zeta h(\zeta) \ln (\zeta)+g(\zeta)$ for some entire functions $g, h-$ see $[\operatorname{Si76},(13)]$. For $x \in \operatorname{supp}(\rho), y \in \operatorname{supp}(\rho)$ and $\lambda$ in a compact set, $\lambda|x-y|$ is uniformly bounded. Hence, there exists a constant $C$ such that for such values of $x, y, \mu, \lambda$,

$$
|F(\lambda|x-y|)-F(\mu|x-y|)| \leq C|\lambda \ln (\lambda)-\mu \ln (\mu)| \cdot|\ln | x-y|| .
$$

The bound $\left|L_{\rho}(\lambda)-L_{\rho}(\mu)\right|_{\mathscr{B}}=O(|\lambda \ln (\lambda)-\mu \ln (\mu)|)$ follows now from Schur's test and from the local integrability of $\ln |x-y|$ on $\mathbb{R}^{2}$.

The operator $L_{\rho}(\lambda)$ belongs to $\mathscr{L}^{2}$ by [Si76, Proposition 3.2]. The second estimate of (2.1) amounts to prove that $\Delta L_{\rho}(\lambda)$ belongs to $\mathscr{B}$, uniformly locally for $\lambda \in K$. By the same argument as in [DyZw16, Theorem 2.1], it suffices to show that $\rho \Delta H_{0}(\lambda) \rho$ belongs to $\mathscr{B}$. Recall that $\Delta \ln |x|=-2 \pi \delta$, so that

$$
\Delta_{x} H_{0}(\lambda, x, y)=\delta(x-y)+\Delta_{x}(F(\lambda|x-y|))
$$

We now compute the Laplacian of $F(\lambda|x-y|)$ with respect to $x$. Note that

$$
\Delta(g(\lambda|z|))=4 \frac{\partial^{2} g(\lambda|z|)}{\partial z \partial \bar{z}}=\frac{\lambda}{|z|} g^{\prime}(\lambda|z|)+\lambda^{2} g^{\prime \prime}(\lambda|z|) .
$$

Define $f(\lambda|z|) \stackrel{\text { def }}{=} \lambda|z| h(\lambda|z|)$. By applying (2.3) to $f \cdot \ln$ instead of $g$, we get

$$
\begin{gathered}
\Delta(f(\lambda|z|) \ln (\lambda|z|))=-2 \pi f(\lambda|z|) \delta+\frac{2 f^{\prime}(\lambda|z|)}{\lambda|z|}+\left(\frac{\lambda f^{\prime}(\lambda|z|)}{|z|}+\lambda^{2} f^{\prime \prime}(\lambda|z|)\right) \ln (\lambda|z|) \\
=\frac{2 f^{\prime}(\lambda|z|)}{|z|}+\left(\frac{\lambda f^{\prime}(\lambda|z|)}{|z|}+\lambda^{2} f^{\prime \prime}(\lambda|z|)\right) \ln (\lambda|z|) .
\end{gathered}
$$

In the second line we used that the product of a smooth function vanishing at 0 with $\delta$ vanishes. The right hand sides of (2.3) and (2.4) both define locally integrable functions of $z \in \mathbb{C}$, uniformly locally in $\lambda$. Since $\rho$ is compactly supported, Schur's test combined with (2.2) shows that $\rho \Delta H_{0}(\lambda) \rho$ belongs to $\mathscr{B}$ uniformly in $\lambda$. This concludes the proof.

Since $L_{\mathscr{V}}(\lambda)$ is in $\mathscr{L}^{2}$, we can define the modified Fredholm determinant $d_{\mathscr{V}}(\lambda)$ by

$$
d_{\mathscr{V}}(\lambda) \stackrel{\text { def }}{=} \operatorname{Det}\left(\operatorname{Id}+\Psi\left(L_{\mathscr{V}}(\lambda)\right)\right), \quad \Psi(z) \stackrel{\text { def }}{=}(1+z) e^{-z}-1, \quad \operatorname{Re}(\lambda) \geq 0,
$$

see [DyZw16, Appendix B]. As $K_{\mathscr{V}}(\lambda)$ is the sum of the rank one operator $\Pi_{\mathscr{V}} \ln (\lambda)$ with $L_{\mathscr{V}}(\lambda), K_{\mathscr{V}}(\lambda)$ belongs to $\mathscr{L}^{2}$. We define

$$
D_{\mathscr{V}}(\lambda) \stackrel{\text { def }}{=} \operatorname{Det}\left(\operatorname{Id}+\Psi\left(K_{\mathscr{V}}(\lambda)\right)\right), \quad \operatorname{Re}(\lambda)>0 .
$$

The negative eigenvalues of $-\Delta+\mathscr{V}$ are exactly the numbers of the form $-\lambda^{2}$, where $\lambda \in(0, \infty)$ is a zero of $D_{\mathscr{V}}(\lambda)$, see [GLMZ05, Theorem 5.4]. The next result is similar to [Si76, Theorem 3.3].

Lemma 2.2. Let $\lambda$ such that $\operatorname{Re} \lambda>0$ and $\operatorname{Id}+L_{V}(\lambda)$ is invertible on $L^{2}$. Then,

$$
D_{\mathscr{V}}(\lambda)=0 \Leftrightarrow 1+\ln (\lambda) \varphi_{\mathscr{V}}(\lambda)=0, \quad \varphi_{\mathscr{V}}(\lambda) \stackrel{\text { def }}{=} \operatorname{Tr}\left(\left(\operatorname{Id}+L_{\mathscr{V}}(\lambda)\right)^{-1} \Pi_{\mathscr{V}}\right) .
$$


If moreover $\lambda \in(0, \infty)$ then $\varphi_{\mathscr{r}}(\lambda) \in \mathbb{R}$.

Proof. To simplify the notations of this proof, we simply write $L_{\mathscr{V}}, K_{\mathscr{V}}$ for the operators $L_{\mathscr{V}}(\lambda), K_{\mathscr{V}}(\lambda)$. Let $\lambda$ with $\operatorname{Re} \lambda>0$ and $\operatorname{Id}+L_{\mathscr{V}}$ invertible on $L^{2}$. Since $K_{\mathscr{V}}=$ $\Pi_{\mathscr{V}} \ln (\lambda)+L_{\mathscr{V}}$ we have

$$
\mathrm{Id}+K_{\mathscr{V}}=\left(\mathrm{Id}+L_{\mathscr{V}}\right)\left(\mathrm{Id}+\left(\operatorname{Id}+L_{\mathscr{V}}\right)^{-1} \Pi_{\mathscr{V}} \ln (\lambda)\right) .
$$

Recall that $1+z=(1+\Psi(z)) e^{z}$ so that for every bounded operator $A$, Id $+A=$ $(\operatorname{Id}+\Psi(A)) e^{A}$. This identity combined with (2.5) shows that

$$
e^{K_{\mathscr{V}}}\left(\operatorname{Id}+\Psi\left(K_{\mathscr{V}}\right)\right)=e^{L_{\mathscr{V}}}\left(\operatorname{Id}+\Psi\left(L_{\mathscr{V}}\right)\right)\left(\operatorname{Id}+\left(\operatorname{Id}+L_{\mathscr{V}}\right)^{-1} \Pi_{\mathscr{V}} \ln (\lambda)\right) .
$$

We observe that $K_{\mathscr{V}}-L_{\mathscr{V}}=\ln (\lambda) \Pi_{\mathscr{V}}$. Since $\Pi_{\mathscr{V}}$ has kernel $-\rho \otimes \mathscr{V} /(2 \pi)$, its trace is equal to $\alpha=-\int_{\mathbb{R}^{2}} \mathscr{V} /(2 \pi)$. Taking the determinant on both sides of (2.6) we obtain

$$
\lambda^{\alpha} D_{\mathscr{V}}(\lambda)=d_{\mathscr{V}}(\lambda) \cdot \operatorname{Det}\left(\operatorname{Id}+\left(\operatorname{Id}+L_{\mathscr{V}}\right)^{-1} \Pi_{\mathscr{V}} \ln (\lambda)\right) .
$$

Since the operator $\left(\mathrm{Id}+L_{\mathscr{Y}}\right)^{-1} \Pi_{\mathscr{Y}} \ln (\lambda)$ is of rank one, the determinant of Id + $\left(\operatorname{Id}+L_{\mathscr{V}}\right)^{-1} \Pi_{\mathscr{V}} \ln (\lambda)$ is equal to $1+\operatorname{Tr}\left(\left(\operatorname{Id}+L_{\mathscr{V}}\right)^{-1} \Pi_{\mathscr{V}} \ln (\lambda)\right)$. Defining $\varphi_{\mathscr{V}}(\lambda)=$ $\operatorname{Tr}\left(\left(\mathrm{Id}+L_{\mathscr{V}}\right)^{-1} \Pi_{\mathscr{V}}\right)$, we obtain

$$
\lambda^{\alpha} D_{\mathscr{V}}(\lambda)=d_{\mathscr{V}}(\lambda) \cdot\left(1+\ln (\lambda) \varphi_{\mathscr{V}}(\lambda)\right) .
$$

Since $\operatorname{Re} \lambda>0$ we have $\lambda \neq 0$ and the first part of the lemma follows.

For the second part of the lemma, it suffices to prove that $D_{\mathscr{V}}(\lambda)$ and $d_{\mathscr{Y}}(\lambda)$ are both real when $\lambda>0$, thanks to (2.7). For such $\lambda, R_{0}(\lambda)$ is selfadjoint. Hence,

$$
\overline{D_{\mathscr{V}}(\lambda)}=\operatorname{Det}\left(\operatorname{Id}+\Psi\left(K_{\mathscr{V}}^{*}\right)\right)=\operatorname{Det}\left(\operatorname{Id}+\Psi\left(\mathscr{V} R_{0}(\lambda) \rho\right)\right) .
$$

Since $\Psi$ vanishes at 0 , there exists $\psi$ entire with $\Psi(z)=z \psi(z)$. Therefore,

$$
\overline{D_{\mathscr{V}}(\lambda)}=\operatorname{Det}\left(\operatorname{Id}+\mathscr{V} R_{0}(\lambda) \rho \psi\left(\mathscr{V} R_{0}(\lambda) \rho\right)\right)=\operatorname{Det}\left(\operatorname{Id}+\rho R_{0}(\lambda) \rho \cdot \psi\left(\mathscr{V} R_{0}(\lambda) \rho\right) \mathscr{V}\right) .
$$

In the last equality, we used that if $B \in \mathscr{B}$ and $A$ is trace-class then $\operatorname{Det}(\operatorname{Id}+$ $B A)=\operatorname{Det}(\mathrm{Id}+A B)$, see [DyZw16, (B.5.13)]. The power series expansion of $\psi(z)=$ $\sum_{m=0}^{\infty} a_{m} z^{m}$ implies that $\rho R_{0}(\lambda) \rho \cdot \psi\left(\mathscr{V} R_{0}(\lambda) \rho\right) \mathscr{V}$

$$
=\rho R_{0}(\lambda) \rho \sum_{m=0}^{\infty} a_{m}\left(\mathscr{V} R_{0}(\lambda) \rho\right)^{m} \mathscr{V}=\sum_{m=0}^{\infty} a_{m}\left(\rho R_{0}(\lambda) \mathscr{V}\right)^{m+1}=\Psi\left(L_{\mathscr{V}}\right) .
$$

Equation (2.8) now shows that $D_{\mathscr{V}}(\lambda)=\overline{D_{\mathscr{V}}(\lambda)}$ when $\lambda \in(0, \infty)$. The same arguments (using that $H_{0}(\lambda)$ is selfadjoint for $\lambda \in(0, \infty)$ ) shows that $d_{\mathscr{V}}(\lambda) \in \mathbb{R}$ if $\lambda \in(0, \infty)$. Hence, (2.7) shows that $\varphi_{\mathscr{V}}(\lambda) \in \mathbb{R}$ when $\lambda \in(0, \infty)$.

\section{Bound state exponentially Close to zero.}

We now focus on the case of a potential $V=V_{\varepsilon}$ given by

$$
V_{\varepsilon}(x) \stackrel{\text { def }}{=} W\left(x, \frac{x}{\varepsilon}\right), \quad W(x, y) \stackrel{\text { def }}{=} \sum_{k \in \mathbb{Z}^{2} \backslash 0} W_{k}(x) e^{i k y} .
$$


Here the functions $W_{k} \in C^{\infty}\left(\mathbb{R}^{2}\right)$ have supports in $\mathbb{B}(0, L)=\left\{x \in \mathbb{R}^{2},|x|<L\right\}$, and $\overline{W_{k}}=W_{-k}$. To simplify notations, we will drop the index $\varepsilon$ in $V_{\varepsilon}$ and write $V=V_{\varepsilon}$. We first investigate the invertibility of $\operatorname{Id}+L_{V}(\lambda)$ for $\varepsilon$ small enough. We will need the following lemma regarding the behavior as $\varepsilon \rightarrow 0$ of certain oscillatory integrals:

Lemma 3.1. The following estimates hold:

$$
|V|_{H^{-2}}=O\left(\varepsilon^{2}\right), \quad\left|\langle D\rangle^{-2} V\langle D\rangle^{-2}\right|_{\mathscr{B}}=O\left(\varepsilon^{2}\right), \quad\langle D\rangle \stackrel{\text { def }}{=}(\operatorname{Id}-\Delta)^{1 / 2} .
$$

Proof. We estimate $|V|_{H^{-2}}$ in a similar way as in the proof of [Dr15, Theorem 1]:

$$
\begin{aligned}
|V|_{H^{-2}} & =\left|\langle\xi\rangle^{-2} \sum_{k \neq 0} \widehat{W}_{k}(\xi-k / \varepsilon)\right|_{2} \leq \sum_{k \neq 0}\left|\langle\xi\rangle^{-2}\langle\xi-k / \varepsilon\rangle^{-2}\langle\xi-k / \varepsilon\rangle^{2} \widehat{W}_{k}(\xi-k / \varepsilon)\right|_{2} \\
& \leq \sum_{k \neq 0}\left|\langle\xi\rangle^{-2}\langle\xi-k / \varepsilon\rangle^{-2}\right|_{\infty} \cdot\left|W_{k}\right|_{H^{2}} \leq C \sum_{k \neq 0}\langle k / \varepsilon\rangle^{-2}\left|W_{k}\right|_{H^{2}}=O\left(\varepsilon^{2}\right) .
\end{aligned}
$$

In the last line we used Peetre's inequality: for $t>0, x, y \in \mathbb{R}^{2}$,

$$
\langle x\rangle^{-t}\langle y\rangle^{-t} \leq 2^{t}\langle x-y\rangle^{-t}
$$

This shows the first estimate.

The boundedness of $\langle D\rangle^{-2} V\langle D\rangle^{-2}$ on $L^{2}$ is equivalent to the boundedness of the multiplication operator by $V$ from $H^{2}$ to $H^{-2}$. We recall that $H^{2}$ is an algebra in dimension 2, and that there exists $C>0$ such that for any $u, f \in H^{2},|f u|_{H^{2}} \leq$ $C|f|_{H^{2}}|u|_{H^{2}}$. The multiplication operator by $u \in H^{2}$ is bounded from $H^{2}$ to $H^{2}$, hence from $H^{-2}$ to $H^{-2}$ with same norm and we deduce the inequality $|f u|_{H^{-2}} \leq$ $C|f|_{H^{-2}}|u|_{H^{2}}$. Applying this with $V=f$ shows that $|V|_{H^{2} \rightarrow H^{-2}} \leq C|V|_{H^{-2}}=O\left(\varepsilon^{2}\right)$. This ends the proof

Lemma 3.2. For every compact subset $K$ of $\{\lambda: \operatorname{Re}(\lambda) \geq 0\}$, there exist $C, \varepsilon_{0}>0$ such that for all $0<\varepsilon \leq \varepsilon_{0}$, the inverse of $\operatorname{Id}+L_{V}(\lambda)$ exist, is given by a convergent Neumann series, and satisfies

$$
\left|\left(\operatorname{Id}+L_{V}(\lambda)\right)^{-1}\right|_{\mathscr{B}} \leq C .
$$

Proof. To prove the lemma it suffices to show that $L_{V}(\lambda)^{2}$ is bounded with $\left|L_{V}(\lambda)^{2}\right|_{\mathscr{B}}<$ $\frac{1}{2}$, for $\varepsilon$ small enough. Recall that $V \in L^{\infty}$; and (Lemma 2.1) that for $\lambda \in K, L_{\rho}(\lambda)$ is uniformly bounded from $L^{2}$ to $H^{2}$ (hence by the adjoint bound, from $H^{-2}$ to $L^{2}$ ). These facts imply

$$
\left|L_{V}(\lambda)^{2}\right|_{\mathscr{B}} \leq C\left|L_{\rho}(\lambda) V L_{\rho}(\lambda)\right|_{\mathscr{B}} \leq C\left|\langle D\rangle^{-2} V\langle D\rangle^{-2}\right|_{\mathscr{B}}
$$

The RHS is $O\left(\varepsilon^{2}\right)$ by Lemma 3.1. This ends the proof.

The negative eigenvalues $-\lambda^{2}$ of the operator $-\Delta+V$ on $L^{2}$ all belong to a fixed compact set: if $-\lambda^{2}$ is a negative eigenvalue, then there exists $0 \neq u \in L^{2}$ such that

$$
-\Delta u+V u+\lambda^{2} u=0 \text {. }
$$


Multiplying by $\bar{u}$ on both sides and integrating by parts, we obtain

$$
|\nabla u|_{2}^{2}+\langle V u, u\rangle_{2}+\lambda^{2}|u|_{2}^{2}=0 .
$$

Thus, $\lambda^{2}|u|_{2}^{2} \leq|V|_{\infty}|u|_{2}^{2}$, which shows that $-\lambda^{2} \in\left[-|V|_{\infty}, 0\right] \subset\left[-M^{2}, 0\right]$ where $M=$ $2+\sum_{k \neq 0}\left|W_{k}\right|_{\infty}$ is independent of $\varepsilon$.

This fact, together with Lemma 2.2 and 3.2 (applied with $K=K_{0} \stackrel{\text { def }}{=}[0, M]$ ), implies that for $\varepsilon$ small enough the set of negative eigenvalues of $-\Delta+V$ is exactly $\left\{-\lambda^{2}\right\}$, where $\lambda$ solves the equation

$$
1+\ln (\lambda) \varphi_{V}(\lambda)=0, \quad \lambda \in K_{0}, \quad \varphi_{V}(\lambda) \stackrel{\text { def }}{=} \operatorname{Tr}\left(\left(\operatorname{Id}+L_{V}(\lambda)\right)^{-1} \Pi_{V}\right) .
$$

Lemma 3.3. Uniformly for $\lambda, \mu$ in $K_{0}=[0, M]$,

$$
\left|\varphi_{V}(\lambda)\right|=O\left(\varepsilon^{2}\right), \quad\left|\varphi_{V}(\lambda)-\varphi_{V}(\mu)\right|=O(|\lambda \ln (\lambda)-\mu \ln (\mu)|) .
$$

Proof. We work exclusively with $\lambda, \mu \in K_{0}$ and $\varepsilon$ small enough so that Lemma 3.2 applies. All the estimates below are uniform for such $\lambda, \varepsilon$. Write $\left(\operatorname{Id}+L_{V}(\lambda)\right)^{-1}=$ $\operatorname{Id}-L_{V}(\lambda)\left(\operatorname{Id}+L_{V}(\lambda)\right)^{-1}$ to get

$$
\begin{gathered}
\varphi_{V}(\lambda)=\operatorname{Tr}\left(\Pi_{V}\right)-\operatorname{Tr}\left(L_{V}(\lambda)\left(\operatorname{Id}+L_{V}(\lambda)\right)^{-1} \Pi_{V}\right)=\frac{-\langle\rho, V\rangle_{2}+\left\langle L_{V}(\lambda) f_{\lambda}, V\right\rangle_{2}}{2 \pi}, \\
f_{\lambda} \stackrel{\text { def }}{=}\left(\operatorname{Id}+L_{V}(\lambda)\right)^{-1} \rho .
\end{gathered}
$$

The term $\langle\rho, V\rangle_{2}$ is clearly independent of $\lambda$ and equal to $O\left(\varepsilon^{\infty}\right)$. Let $P_{k}=\left(k_{1} D_{x_{1}}+\right.$ $\left.k_{2} D_{x_{2}}\right) /|k|^{2}$, so that $P_{k} e^{i k x / \varepsilon}=\varepsilon e^{i k x / \varepsilon}$. Since $P_{k}$ is selfadjoint, $\left\langle L_{V}(\lambda) f_{\lambda}, V\right\rangle_{2}=$

$$
\varepsilon^{2} \sum_{k \neq 0}\left\langle W_{k} \cdot L_{V}(\lambda) f_{\lambda}, \rho P_{k}^{2} e^{-i k \bullet / \varepsilon}\right\rangle_{2}=\varepsilon^{2} \sum_{k \neq 0}\left\langle P_{k}^{2}\left(W_{k} \cdot L_{V}(\lambda) f_{\lambda}\right), \rho e^{-i k \bullet / \varepsilon}\right\rangle_{2} .
$$

The Cauchy-Schwarz inequality yields

$$
\left|\varphi_{V}(\lambda)\right| \leq O\left(\varepsilon^{\infty}\right)+C \varepsilon^{2} \sum_{k \neq 0}\left|P_{k}^{2}\left(W_{k} \cdot L_{V}(\lambda) f_{\lambda}\right)\right|_{2} .
$$

By Lemma 3.2, the operator ( $\left.\operatorname{Id}+L_{V}(\lambda)\right)^{-1}$ is uniformly bounded in $\mathscr{B}$, hence $\left|f_{\lambda}\right|_{2}=$ $O(1)$. Since $L_{V}(\lambda)$ maps $L^{2}$ to $H^{2}$ the operator $P_{k}^{2} W_{k} L_{V}(\lambda)$ maps $L^{2}$ to $L^{2}$ with

$$
\left|P_{k}^{2} W_{k} L_{V}(\lambda)\right|_{\mathscr{B}} \leq C \frac{\left|W_{k}\right|_{C^{2}}}{|k|^{2}}|V|_{\infty}
$$

Thus $\left|\varphi_{V}(\lambda)\right|=O\left(\varepsilon^{2}\right)$ as claimed.

Regarding the second estimate in (3.2), we use (3.3) and the Cauchy-Schwarz inequality to see that

$$
\begin{gathered}
\left|\varphi_{V}(\lambda)-\varphi_{V}(\mu)\right| \leq \frac{1}{2 \pi}\left|L_{V}(\lambda) f_{\lambda}-L_{V}(\mu) f_{\mu}\right|_{2}|V|_{2} \\
\leq C\left|L_{\rho}(\lambda)-L_{\rho}(\mu)\right|_{\mathscr{B}}+C\left|f_{\lambda}-f_{\mu}\right|_{2} \leq C\left|L_{\rho}(\lambda)-L_{\rho}(\mu)\right|_{\mathscr{B} .}
\end{gathered}
$$

In the above we used $f_{\lambda}=\left(\operatorname{Id}+L_{V}(\lambda)\right)^{-1} \rho$, the resolvent identity, and the uniform boundedness of $\left(\operatorname{Id}+L_{V}(\lambda)\right)^{-1}$. The conclusion follows now from Lemma 2.1. 
We now identify $\lim _{\varepsilon \rightarrow 0} \varepsilon^{-2} \varphi_{V}(\lambda)$ :

Lemma 3.4. Uniformly for $\lambda \in K_{0}$,

$$
\varphi_{V}(\lambda)=\frac{\varepsilon^{2}}{2 \pi} \int_{\mathbb{R}^{2}} \Lambda_{0}(x) d x+o\left(\varepsilon^{2}\right), \quad \Lambda_{0}(x) \stackrel{\text { def }}{=} \sum_{k \neq 0} \frac{\left|W_{k}(x)\right|^{2}}{|k|^{2}} .
$$

Proof. 1. We claim that it is enough to show (3.4) for $\lambda \in[1,2]$. Indeed, Lemma 3.3 shows that $\varepsilon^{-2} \varphi_{V}$ is uniformly bounded. In addition, it is a holomorphic function of $\lambda \in K_{0}$. Hence, after possibly passing to a subsequence, it converges uniformly locally to a holomorphic function. If (3.4) holds for $\lambda \in[1,2], \varepsilon^{-2} \varphi_{V}$ has only one accumulation point on $[1,2]$, hence - by the unique continuation principle - only one accumulation point on $K_{0}$. This would prove that (3.4) holds uniformly on $K_{0}$. Below we show (3.4) for $\lambda \in[1,2]$. We will always assume that $\varepsilon$ is small enough so that Lemma 3.2 holds.

2. By expanding $\left(\operatorname{Id}+L_{V}(\lambda)\right)^{-1}$ into a finite Born series,

$$
\begin{gathered}
\varphi_{V}(\lambda)=\operatorname{Tr}\left(\Pi_{V}\right)-\operatorname{Tr}\left(L_{V}(\lambda) \Pi_{V}\right)+\operatorname{Tr}\left(L_{V}(\lambda)\left(\operatorname{Id}+L_{V}(\lambda)\right)^{-1} L_{V}(\lambda) \Pi_{V}\right) \\
=-\frac{1}{2 \pi} \int_{\mathbb{R}^{2}} V(x) d x+\frac{1}{2 \pi}\left\langle L_{V}(\lambda) \rho, V\right\rangle_{2}-\frac{1}{2 \pi}\left\langle\left(\operatorname{Id}+L_{V}(\lambda)\right)^{-1} L_{V}(\lambda) \rho, L_{V}(\lambda)^{*} V\right\rangle_{2} .
\end{gathered}
$$

The first term is $O\left(\varepsilon^{\infty}\right)$. We note that since $\lambda \in[1,2], L_{V}(\lambda)^{*}=L_{V}(\lambda)$ and the third term can be bounded using the Cauchy-Schwarz inequality:

$$
\left|\left\langle\left(\operatorname{Id}+L_{V}(\lambda)\right)^{-1} L_{V}(\lambda) \rho, L_{V}(\lambda) V\right\rangle_{2}\right| \leq\left|\left(\operatorname{Id}+L_{V}(\lambda)\right)^{-1}\right|_{\mathscr{B}}\left|L_{V}(\lambda) \rho\right|_{2}\left|L_{V}(\lambda) V\right|_{2}
$$

We note that $L_{V}(\lambda) \rho=L_{\rho}(\lambda) V$ and we recall that $L_{V}(\lambda)$ is bounded from $H^{-2}$ to $L^{2}$. Hence Lemma 3.1 implies that $\left|L_{V}(\lambda) \rho\right|_{2} \leq C|V|_{H^{-2}}=O\left(\varepsilon^{2}\right)$. Similarly,

$$
\left|L_{V}(\lambda) V\right|_{2} \leq C\left|L_{\rho}(\lambda) V\right|=O\left(\varepsilon^{2}\right)
$$

This proves that the RHS of $(3.5)$ is $O\left(\varepsilon^{4}\right)$. The steps below are devoted to estimating the leading term in the expansion of $\varphi_{V}(\lambda):\left\langle L_{V}(\lambda) \rho, V\right\rangle_{2}$.

3. Using $L_{V}(\lambda)=K_{V}(\lambda)-\Pi_{V} \ln (\lambda)$, we have uniformly on $[1,2]$

$$
\begin{aligned}
\left\langle L_{V}(\lambda) \rho, V\right\rangle_{2} & =\int_{\mathbb{R}^{2} \times \mathbb{R}^{2}} R_{0}(\lambda, x, y) V(y) V(x) d x d y+\frac{\ln (\lambda)}{2 \pi}\left(\int_{\mathbb{R}^{2}} V(x) d x\right)^{2} \\
& =\int_{\mathbb{R}^{2} \times \mathbb{R}^{2}} \phi_{\lambda}(x-y) V(y) V(x) d x d y+O\left(\varepsilon^{\infty}\right) .
\end{aligned}
$$

Here the function $\phi_{\lambda}$ has Fourier transform equal to $\widehat{\phi_{\lambda}}(\xi)=\left(|\xi|^{2}+\lambda^{2}\right)^{-1}$. The identity (3.6) and the Plancherel formula show that $\left\langle L_{V}(\lambda) \rho, V\right\rangle_{2}$ is equal modulo $O\left(\varepsilon^{\infty}\right)$ to

$$
\left.\widehat{\left\langle\phi_{\lambda}\right.} \widehat{V}, \widehat{V}\right\rangle_{2}=\int_{\mathbb{R}^{2}} \frac{\widehat{V}(\xi) \widehat{V}(-\xi)}{|\xi|^{2}+\lambda^{2}} d \xi
$$


As the Fourier transform of $V$ is given by $\sum_{k \neq 0} \widehat{W}_{k}(\xi-k / \varepsilon)$ we obtain

$$
\left\langle L_{V}(\lambda) \rho, V\right\rangle_{2}=\sum_{k, \ell} I\left[W_{k}, W_{\ell}\right]+O\left(\varepsilon^{\infty}\right), \quad I\left[W_{k}, W_{\ell}\right] \stackrel{\text { def }}{=} \int_{\mathbb{R}^{2}} \frac{\widehat{W_{k}}(\xi-k / \varepsilon) \widehat{W_{\ell}}(-\xi+\ell / \varepsilon)}{|\xi|^{2}+\lambda^{2}} d \xi .
$$

We will study $I\left[W_{k}, W_{\ell}\right]$ depending whether $k+\ell \neq 0$ or $k+\ell=0$.

4. Assume that $k+\ell \neq 0$. We have

$$
\begin{gathered}
\left|I\left[W_{k}, W_{\ell}\right]\right| \leq C\left|W_{k}\right|_{C^{3}}\left|W_{\ell}\right|_{C^{3}} \int_{\mathbb{R}^{2}}\langle\xi\rangle^{-2}\langle\xi-k / \varepsilon\rangle^{-3}\langle-\xi+\ell / \varepsilon\rangle^{-3} d \xi \\
\quad \leq C \varepsilon^{5 / 2} \frac{\left|W_{k}\right|_{C^{3}}\left|W_{\ell}\right|_{C^{3}}}{|k-\ell|^{5 / 2}} \int_{\mathbb{R}^{2}}\langle\xi\rangle^{-2}\langle\xi-k / \varepsilon\rangle^{-1 / 2}\langle-\xi+\ell / \varepsilon\rangle^{-1 / 2} d \xi
\end{gathered}
$$

To get the second line, we controlled $\langle\xi-k / \varepsilon\rangle^{-5 / 2}\langle-\xi+\ell / \varepsilon\rangle^{-5 / 2}$ by $\langle(k-\ell) / \varepsilon\rangle^{-5 / 2}$ with Peetre's inequality (3.1). Hölder's inequality yields

$$
\left|I\left[W_{k}, W_{\ell}\right]\right| \leq C \varepsilon^{5 / 2} \frac{\left|W_{k}\right|_{C^{3}}\left|W_{\ell}\right|_{C^{3}}}{|k-\ell|^{5 / 2}}
$$

5. We now focus on the case $k+\ell=0$. A substitution yields

$$
I\left[W_{k}, W_{-k}\right]=\varepsilon^{2} \int_{\mathbb{R}^{2}} \frac{\widehat{W_{k}}(\xi) \widehat{W_{-k}}(-\xi)}{|\varepsilon \xi+k|^{2}+(\varepsilon \lambda)^{2}} d \xi .
$$

We split the domain of integration $\mathbb{R}^{2}$ into two parts: $\mathbb{B}\left(0, \varepsilon^{-3 / 4}\right)$ and $\mathbb{R}^{2} \backslash \mathbb{B}\left(0, \varepsilon^{-3 / 4}\right)$. If $\xi \in \mathbb{R}^{2} \backslash \mathbb{B}\left(0, \varepsilon^{-3 / 4}\right)$ then $|\varepsilon \xi+k|+(\varepsilon \lambda)^{2} \geq(\varepsilon \lambda)^{2} \geq \varepsilon^{2}$ since $\lambda \in[1,2]$. Therefore

$$
\begin{gathered}
\left|\int_{\mathbb{R}^{2} \backslash \mathbb{B}\left(0, \varepsilon^{-1 / 2}\right)} \frac{\widehat{W_{k}}(\xi) \widehat{W_{-k}}(-\xi)}{|\varepsilon \xi+k|^{2}+(\varepsilon \lambda)^{2}} d \xi\right| \leq \int_{|\xi| \geq \varepsilon^{-3 / 4}} \frac{1}{\varepsilon^{2}}\left|\widehat{W}_{k}(\xi) \widehat{W_{-k}}(-\xi)\right| d \xi \\
\leq C \int_{|\xi| \geq \varepsilon^{-3 / 4}} \frac{1}{\varepsilon^{2}}\langle\xi\rangle^{-6}\left|W_{k}\right|_{C^{3}}\left|W_{-k}\right|_{C^{3}} d \xi \leq C \varepsilon\left|W_{k}\right|_{C^{3}}\left|W_{-k}\right|_{C^{3}} .
\end{gathered}
$$

We now assume that $\xi \in \mathbb{B}\left(0, \varepsilon^{-3 / 4}\right)$, hence $|\varepsilon \xi+k| \geq|k|-\varepsilon^{1 / 4} \geq|k| / 2$. This implies

$$
\frac{1}{|\varepsilon \xi+k|^{2}+(\varepsilon \lambda)^{2}}-\frac{1}{|k|^{2}}=\frac{-(\varepsilon \lambda)^{2}-\varepsilon^{2}|\xi|^{2}-2\langle\varepsilon \xi, k\rangle}{|k|^{2}\left(|\varepsilon \xi+k|^{2}+(\varepsilon \lambda)^{2}\right)}=O\left(\varepsilon^{1 / 4}|k|^{-3}\right) .
$$

This implies

$$
\begin{gathered}
\int_{\mathbb{B}\left(0, \varepsilon^{-1 / 2}\right)} \frac{\widehat{W_{k}}(\xi) \widehat{W_{-k}}(-\xi)}{|\varepsilon \xi+k|^{2}+(\varepsilon \lambda)^{2}} d \xi=\int_{\mathbb{B}\left(0, \varepsilon^{-1 / 2}\right)} \frac{\widehat{W_{k}}(\xi) \widehat{W_{-k}}(-\xi)}{|k|^{2}} d \xi+O\left(\varepsilon^{1 / 4}\right) \frac{\left|W_{k}\right|_{C^{2}}\left|W_{-k}\right|_{C^{2}}}{|k|^{3}} \\
=\int_{\mathbb{R}^{2}} \frac{\widehat{W_{k}}(\xi) \widehat{W_{-k}}(-\xi)}{|k|^{2}} d \xi+O\left(\varepsilon^{1 / 4}\right) \frac{\left|W_{k}\right|_{C^{2}}\left|W_{-k}\right|_{C^{2}}}{|k|^{3}} .
\end{gathered}
$$


6. Combine the results of steps 1-5 to get

$$
\begin{aligned}
& \varepsilon^{-2} \varphi_{V}(\lambda)=\frac{1}{2 \pi} \sum_{k \neq 0} I\left[W_{k}, W_{-k}\right]+o(1)=\frac{1}{2 \pi} \sum_{k \neq 0} \frac{1}{|k|^{2}} \int_{\mathbb{R}^{2}} \widehat{W_{k}}(\xi) \widehat{W_{-k}}(-\xi) d \xi+o(1) \\
& =\frac{1}{2 \pi} \sum_{k \neq 0} \frac{1}{|k|^{2}}\left\langle W_{k}, \overline{W_{-k}}\right\rangle_{2}+o(1)=\frac{1}{2 \pi} \int_{\mathbb{R}^{2}} \Lambda_{0}(x) d x+o(1), \quad \Lambda_{0}(x)=\sum_{k \neq 0} \frac{\left|W_{k}(x)\right|^{2}}{|k|^{2}} .
\end{aligned}
$$

In the above we used that as $V$ is real-valued, $W_{k}=\overline{W_{-k}}$. This estimate completes the proof of the lemma.

We finally turn to the proof of Theorem 1.

Proof of Theorem 1. By the discussion following the proof of Lemma 3.2, for $\varepsilon$ small enough the negative eigenvalues of $-\Delta+V$ are all in a fixed compact set and are the negative squares of the resonances of $V$ in $K_{0}$. We now work for $\lambda \in K_{0}$.

For $\varepsilon$ small enough, Id $+L_{V}(\lambda)$ is invertible by Lemma 3.2. Lemma 2.2 shows that the resonances of $V$ are the zeros of $1+\ln (\lambda) \varphi_{V}(\lambda)$. Since $\varphi_{V}(\lambda)=\frac{\varepsilon^{2}}{2 \pi} \int_{\mathbb{R}^{2}} \Lambda_{0}+o\left(\varepsilon^{2}\right)$ uniformly on $K_{0}=[0, M]$, if $\varepsilon$ is small enough then

$$
\lim _{\lambda \rightarrow 0} 1+\ln (\lambda) \varphi_{V}(\lambda)=-\infty, \quad 1+\ln (M) \varphi_{V}(M) \geq \frac{1}{2} .
$$

We recall that $\varphi_{V}$ is real-valued on $K_{0}$. The intermediate value theorem shows that $1+\ln (\lambda) \varphi_{V}(\lambda)$ has at least one zero $\lambda$ on $[0, M]$. It must satisfy

$$
\ln (\lambda)=-\frac{1}{\varphi_{V}(\lambda)}=-\frac{2 \pi}{\varepsilon^{2} \int_{\mathbb{R}^{2}} \Lambda_{0}(x) d x+o\left(\varepsilon^{2}\right)},
$$

or equivalently,

$$
\lambda=\exp \left(-\frac{2 \pi}{\varepsilon^{2} \int_{\mathbb{R}^{2}} \Lambda_{0}(x) d x+o\left(\varepsilon^{2}\right)}\right) .
$$

It remains to show that $\lambda$ is the unique resonance of $V$ in $K_{0}$ for $\varepsilon$ small enough. We argue as in [Si76, Theorem 2.3]. If $\mu \neq \lambda$ is another resonance in $K_{0}$ then $1+$ $\ln (\mu) \varphi_{V}(\mu)=0$. Hence,

$$
\left|\frac{1}{\ln (\mu)}-\frac{1}{\ln (\lambda)}\right|=\left|\varphi_{V}(\mu)-\varphi_{V}(\lambda)\right| \text {. }
$$

In addition, for $\varepsilon$ small enough, $|\ln (\lambda)|^{-1} \geq c \varepsilon^{2}$ and $|\ln (\mu)|^{-1} \geq c \varepsilon^{2}$. Hence, a lower bound for the right hand side of $(3.8)$ is

$$
\left|\frac{1}{\ln (\mu)}-\frac{1}{\ln (\lambda)}\right| \geq \frac{1}{|\ln (\lambda)||\ln (\mu)|}\left|\int_{\lambda}^{\mu} \frac{d t}{t}\right| \geq \frac{|\lambda-\mu|}{|\ln (\lambda)||\ln (\mu)||\lambda+\mu|} \geq \frac{c^{2} \varepsilon^{4}|\lambda-\mu|}{|\lambda+\mu|} .
$$

An upper bound for the left hand side of (3.8) is provided by Lemma 3.3: $\mid \varphi_{V}(\lambda)-$ $\varphi_{V}(\mu)|\leq C| \lambda \ln (\lambda)-\mu \ln (\mu) \mid$. Therefore, (3.8) implies

$$
\frac{c^{2} \varepsilon^{4}}{|\lambda+\mu|} \leq C \frac{|\lambda \ln (\lambda)-\mu \ln (\mu)|}{|\lambda-\mu|} .
$$


Since the derivative of $t \ln (t)$ is $1+\ln (t)$, and since $\lambda, \mu$ remain in a bounded set with $\ln (\lambda)=O\left(\varepsilon^{-2}\right)$ (and a similar bound for $\mu$ ), we deduce that $\varepsilon^{4} \leq C \varepsilon^{-2}|\lambda+\mu|$. In addition, $|\ln (\lambda)|^{-1} \geq c \varepsilon^{2}$ implies $|\lambda| \leq e^{-c^{-1} \varepsilon^{-2}}$ (and a similar bound for $\mu$ ). Hence, the existence of $\mu$ implies $c^{2} \varepsilon^{4} \leq 2 C \varepsilon^{-2} e^{-c^{-1} \varepsilon^{-2}}=O\left(\varepsilon^{\infty}\right)$. This is a contradiction.

\section{REFERENCES}

[Bo07] D. I. Borisov, Some singular perturbations of periodic operators. Theoret. and Math. Phys. 151(2) (2007), 614-624.

[BoGa06] D. I. Borisov and R. R. Gadyl'shin, On the spectrum of the Schrödinger operator with a rapidly oscillating compactly supported potential. Theoret. and Math. Phys. 147 (2006), no. 1, 496-500.

[Ch06] T. Christiansen, Schrödinger operators with complex-valued potentials and no resonances. Duke Math Jour. 133 (2006), 313-323.

[Di16] M. Dimassi, Semi-classical asymptotics for Schrödinger operator with oscillating decaying potential. To appear in Canad. Math. Bull.

[Dr15] A. Drouot, Scattering resonances for highly oscillatory potentials. Preprint, arXiv:1509.04198.

[DR16] V. Duchêne and N. Raymond, Spectral asymptotics for the Schrödinger operator on the line with spreading and oscillating potentials. Preprint, arXiv:1609.01990.

[DVW14] V. Duchêne, I. Vukićević and M. I. Weinstein, Scattering and localization properties of highly oscillatory potentials. Comm. Pure Appl. Math. 67 (2014), no. 1, 83-128.

[DW11] V. Duchêne and M. I. Weinstein, Scattering, homogenization, and interface effects for oscillatory potentials with strong singularities. Multiscale Model. Simul. 9 (2011), no. 3, 1017-1063.

[DyZw16] S. Dyatlov and M. Zworski, Mathematical theory of scattering resonances. Lecture notes available online.

[GLMZ05] F. Gesztesy, Y. Latushkin, M. Mitrea and M. Zinchenko, Nonselfadjoint operators, infinite determinants, and some applications. Russ. J. Math. Phys. 12 (2005), no. 4, 443-471.

[Si76] B. Simon, The bound state of weakly coupled Schrödinger operators in one and two dimensions. Ann. Physics 97 (1976), no. 2, 279-288.

E-mail address: alexis.drouot@gmail.com 